\title{
Piloting a problem solving module for undergraduate mathematics students
}

David McConnell, School of Mathematics, Cardiff University, Cardiff, UK. Email: mcconnelld@cardiff.ac.uk

\section{Abstract}

We report on a new problem solving module for second-year undergraduate mathematics students first piloted during the 2016-17 academic year at Cardiff University. This module was introduced in response to the concern that for many students, traditional teaching and assessment practices do not offer sufficient opportunities for developing problem-solving and mathematical thinking skills, and more generally, to address the recognised need to incorporate transferrable skills into our undergraduate programmes. We discuss the pedagogic and practical considerations involved in the design and delivery of this module, and in particular, the question of how to construct open-ended problems and assessment activities that promote mathematical thinking, and reward genuinely original and independent mathematical work.

Keywords: Problem solving, mathematical thinking, employability, transferrable skills.

\section{Motivation}

The ability to apply mathematical knowledge to tackle unfamiliar problems is widely recognised as an essential skill that all mathematics graduates should possess (QAA, 2015; Badger et al., 2012). However, there is widespread concern that for many undergraduates, 'traditional' teaching and assessment methods do not offer sufficient opportunities for developing these skills (Rowlett, 2011, p.19).

Given that exams are the main method of summative assessment in mathematics (lannone and Simpson, 2012), it is natural that much of students' independent study time is spent preparing for them. However, in most exams, time constraints make it impossible to ask students to solve genuinely challenging problems, and further, there is limited scope for rewarding original and interesting mathematical work. While regular exercise sheets and textbook problems may provide an avenue for assigning more difficult work in many modules, not all students engage with these in the way we might like - anecdotally, it would appear that many view the problems as templates for future exam questions, and use them (together with model answers, if these are provided) simply as a revision tool closer to exam time. As a consequence, the problems that students encounter are often predictable and narrow in focus, and there is a risk that students may graduate with reasonably good grades without important mathematical skills being developed (Selden, Selden and Mason, 1994).

In addition to these mathematical considerations, the need to integrate more general transferrable skills into undergraduate mathematics programmes is also widely acknowledged (Challis, Houston and Stirling, 2003, pp.14-17; Waldock, 2011). For example, while good mathematical writing is encouraged, this is often overlooked when marking exams, and students are rarely asked to produce more substantial pieces of written work. The ability to communicate mathematical work orally has been identified as another area of weakness. Collaboration and group working, while encouraged by many lecturers, is also rarely formally embedded in modules and their assessment. In light of the current institutional and regulatory focus on employability in UK Higher Education, it was a challenge to not only develop these skills, but importantly, to do so in a meaningful way within the context of university-level mathematics. 


\section{Designing a new module}

In response to the concerns raised in the previous section, a new module entitled Problem Solving was introduced in the 2016-17 academic year. This is a second year (level 5), 10 credit module, which has been piloted as an optional module, available to all single honours mathematics students on our undergraduate programmes (including integrated masters' students). .

The decision to introduce Problem Solving as a second-year module was partly motivated by research that has indicated that many students become significantly disengaged with their studies during the second-year of their undergraduate programme, and often do not recover from this (Croft and Grove, 2015). Moreover, we wanted to students to attempt problems that made use of the results and methods from their first-year modules, thus it was necessary for them to have encountered enough university-level mathematics beforehand.

In this module, there would be very little formal teaching, and no new mathematical content - students would spend the majority of class time working on unseen, open-ended problems using their existing mathematical knowledge. This work would be carried out in small groups, which would remain fixed throughout the module. Finally, the module would be assessed entirely through (group) reports and presentations, and the marking criteria would reward evidence of genuinely original mathematical thinking, rather than simply results.

Contact time for Problem Solving consists of a single three-hour block per week. The first week is an introductory session, explaining the aim and structure of the module. Most of the remaining classes are tutorial-style 'problem solving sessions' where students are presented with a choice of two unfamiliar problems, which they work on during class and submit a short summary of their work at the end.

A major part of the lecturer's role during these sessions is to advise students on how to work independently, rather than depending on the practice of imitating similar work. For example, when students are struggling, this may involve suggesting questions to investigate to gain insight into the problem at hand, as an alternative to searching for solutions on the internet. Equally important is to reassure students that when doing mathematics, much time is spent being stuck, confused, uncertain, or simply wrong, and that this is not a sign of failure, but a necessary step along the way.

Half-way through the semester, there is a peer-assessment session where groups exchange drafts of their work and give feedback. During this time, we also discuss how students might demonstrate evidence of intelligent problem solving strategies, with reference to the ideas in Polya (1945), and Mason, Burton and Stacey (2011). During the final two weeks of the semester, students give group presentations on a problem of their choice.

\section{What makes a good problem?}

The most challenging task when designing this module was creating a set of suitable problems, specifically problems that demand aspects of mathematical work that are typically overlooked in other modules, while at the same time make use of results from our undergraduate mathematics curriculum. Although problems from similar courses being taught elsewhere can be found in the literature, such as in Badger et al. (2012), we found that many of them did not offer sufficient opportunity to make use of university-level mathematics, particularly topics in pure mathematics. Based on some of the ideas discussed in Badger et al. (2012, p.27) and Mason (2000, pp.104-105), we derived properties that we suggest such problems should have, which we discuss here.

Unlike the questions that students encounter on exercise sheets and exams, most mathematical work, both in academic research and elsewhere, does not begin with a precise question and end with an elegant, complete, and correct solution, but instead involves an iterative process of examining 
examples, restricting to special cases, making, testing, and refining conjectures, and many other activities (Hirst and Biggs, 1969). By contrast, when working on exercise sheets, the student typically knows from the outset that the problem does indeed have a solution, and moreover, that the lecturer expects that they ought to able to find it. Rarely are students assigned problems that are ambiguous, open-ended, or where they could only ever be expected to arrive at a partial solution.

For this reason, we tried to design problems that both required some effort to translate into a precise mathematical question or questions, and admit many possible routes towards a solution, and ideally, some ambiguity as to what constitutes a valid solution. Some degree of evaluation of one's own work should be required, which may take many forms - testing examples, looking for a more elegant solution, seeking generalisations (or showing that they cannot be found).

Given that an exercise sheet is associated with a particular module, it is usually clear from the beginning that the necessary tools for solving these problems will be found mostly within the syllabus. Again, this is somewhat artificial, and we tried to address this by designing problems that were not tied to any particular topic, and where students were free to research new material as part of the process of solving it.

A major difficulty with this approach was the question of how to deal with students with different abilities. Indeed, a reasonably challenging problem for one student may be a trivial exercise for another, and completely impossible for a third. To account for this, we tried to design problems that would allow any reasonable student to reach a 'partial solution' (e.g. covering a simplified analogy or elementary special cases of the problem), and moreover, had significant scope for generalisation that a stronger student could undertake.

\section{Example problems}

In this section we give some examples of the problems assigned to students in this module. Broadly speaking, these fell into two categories - problems from pure mathematics, and coding problems (all students take a Python course in year one).

The first example of a purely mathematical problem was adapted from Mason, Burton and Stacey (2011).

Problem 1: Let $S$ be the set of those functions $f: R \rightarrow R$ having the property that, for all intervals $[a, b]$, the mean value of $f^{\prime}$ over the interval $[a, b]$ occurs at the midpoint. Identify some suitable conditions that can be used to determine whether or not a given function belongs to $S$.

Problem 1 reflects a common theme in research mathematics - trying to obtain a useful classification of objects satisfying a certain property. There is significant uncertainty involved for the student in determining what might constitute a 'suitable condition that can be used' - indeed, it is necessary to experiment with many examples before making a conjecture about what a 'solution' to this problem might look like.

Once they have made a suitable conjecture, an average student should manage to answer this question completely for polynomials using some fairly elementary algebra, while going beyond this demands the use of more advanced tools (e.g. Taylor's Theorem). For a student who does answer this question more or less completely, many interesting generalisations may be prompted - for example, is there an analogy of this property in higher dimensions? Are there functions $f$ for which the mean value of $f^{\prime}$ over $[a, b]$ always occurs at a point that divides the interval $[a, b]$ according to another fixed ratio?

The second example is a typically example of one of the coding problems in this module. 
Problem 2: Approximating $\pi$. Using as many approaches as you can think of, try to construct some algorithms/formulae that can be used to approximate $\pi$ using only integers and the operations of addition, subtraction, multiplication and division. Evaluate and compare any successful approaches you have found, and explain the difficulties that arose in your unsuccessful attempts.

At a first glance, this problem looks quite straightforward, until students realise that most obvious approximations of $\pi$ make extensive use of irrational numbers e.g. using the perimeter or area of a polygon to approximate that of a circle. A significant amount of work is needed to find any suitable algorithms. Some interesting examples of student work on this problem used Taylor Series for inverse trigonometric functions, and various methods of numerical integration to estimate the area of a circle.

Some problems lead to some genuinely impressive work being submitted by students, such as the following, also adapted from Mason, Burton and Stacey (2011).

Problem 3: A point $(x, y) \in R^{2}$ is called rational if both $x$ and $y$ are rational numbers. For a curve $C$ in $R^{2}$, let $N(C)$ denote the (possibly infinite) number of rational points that lie on $C$. Determine the possible values that $N(C)$ can take for various types of curve $C$ (e.g. lines, circles, parabolas). For each value of $N(C)$ that you find, you should attempt to describe the set of all curves of this type that have exactly this number of rational points (e.g. a circle $C$ has $N(C)=1$ if and only if ...).

Not all problems assigned to students in this module have been a success. Our final example was adapted from the Industrial Problem Solving for Higher Education (University of Bristol, 2016) website.

Problem 4: As part of a manufacturing process, it is necessary to cut discs of radius $12.5 \mathrm{~cm}$, and discs of radius $5 \mathrm{~cm}$, from square sheets of steel of size $1 \mathrm{~m}^{2}$. They need twice the number of small disks as they do large disks. You job is to identify the best arrangement of cutting heads to minimise the amount of wasted steel. Design and test (using Python) some algorithms for finding the optimal arrangement of the discs, and compare their performance.

This looks like a reasonable problem at first - there are many natural initial steps that can be taken, and a large number of potential approaches that students could undertake. However, on closer inspection, most strategies that a year 2 student could reasonably be expected to pursue either lack non-trivial mathematical content (such as using basic geometry and examining arrangements by inspection), or else are far too difficult. Problem 4 illustrates the need for the lecturer to test problems extensively for suitability before assigning them.

\section{Assessment}

In the 2016-17 academic year, Problem Solving consisted of 11 three-hour classes, one per week. During 9 of those classes, students were assigned a new problem at the start of each class, and spent the class working on that weeks' problem in groups. For the summative coursework, each group was required to submit a total of 18 pieces of work, consisting of:

- a short summary of their work, handed up at the end of each class, worth $5 \%$ of their grade, and;

- a more detailed report (typeset in LaTeX) prepared over the following week, worth another $5 \%$ of their grade.

Note that all reports were group reports, and each member received the same grade irrespective of contribution. The remaining $10 \%$ of their grade came from a group presentation on one of their reports. 
In the written reports, rather than simply presenting their solutions, students are instructed to outline the steps that they took, and their reasons for taking these steps, as well as evaluating any results that they obtained. Credit is awarded for demonstrating an intelligent problem-solving strategy, rather than simply the progress made towards a complete solution. The hope is that by writing about the process of doing mathematics, students will recognise general themes in mathematical work, and strategies that one might undertake in attacking an unfamiliar problem. Moreover, they are encouraged to pursue any interesting related questions that are prompted by their work, even if not directly relevant to the problem statement - students are rewarded for any genuinely original work that contributes to their reports.

Identifying and preventing academic malpractice is a significant concern in written coursework in mathematics (Challis, Houston and Stirling, 2003, pp.23-25), particularly since solutions for almost any conceivable mathematical problem can be found on the internet. We did not aim to restrict the use of external resources, but instead to set assessment criteria that discourage the practice of imitating similar work. By assigning ambiguous, open-ended problems, and by requiring that students justify and evaluate their work, we believe that any unfair advantage derived from finding solutions to similar problems is minimised.

\section{Evaluation and future outlook}

Enrolment in Problem Solving was initially capped at 32 students in 2016-17, though it is now available as an optional module for all second year undergraduates in the School of Mathematics. A total of 25 students took Problem Solving in 2016-17, followed by 39 students in 2017-18, out of a total of approximately 160 second year students. In both years, just over $40 \%$ of those enrolled in the module have been female, which is broadly in line with the gender ratio across the School of Mathematics.

Some interesting patterns emerge when comparing those students who took Problem Solving to their cohort as a whole. In both 2016-17 and 2017-18, those students who chose Problem Solving in year 2 had had a significantly lower year 1 average than those who did not (see Figure 1). One possible explanation for this is that a module that is assessed entirely with coursework may appeal to students who under-perform in written exams. In the medium term, it will be interesting to study how these 
students perform over the course of their degrees, and in particular, to investigate whether or not choosing Problem Solving is associated with any improvements in academic performance.

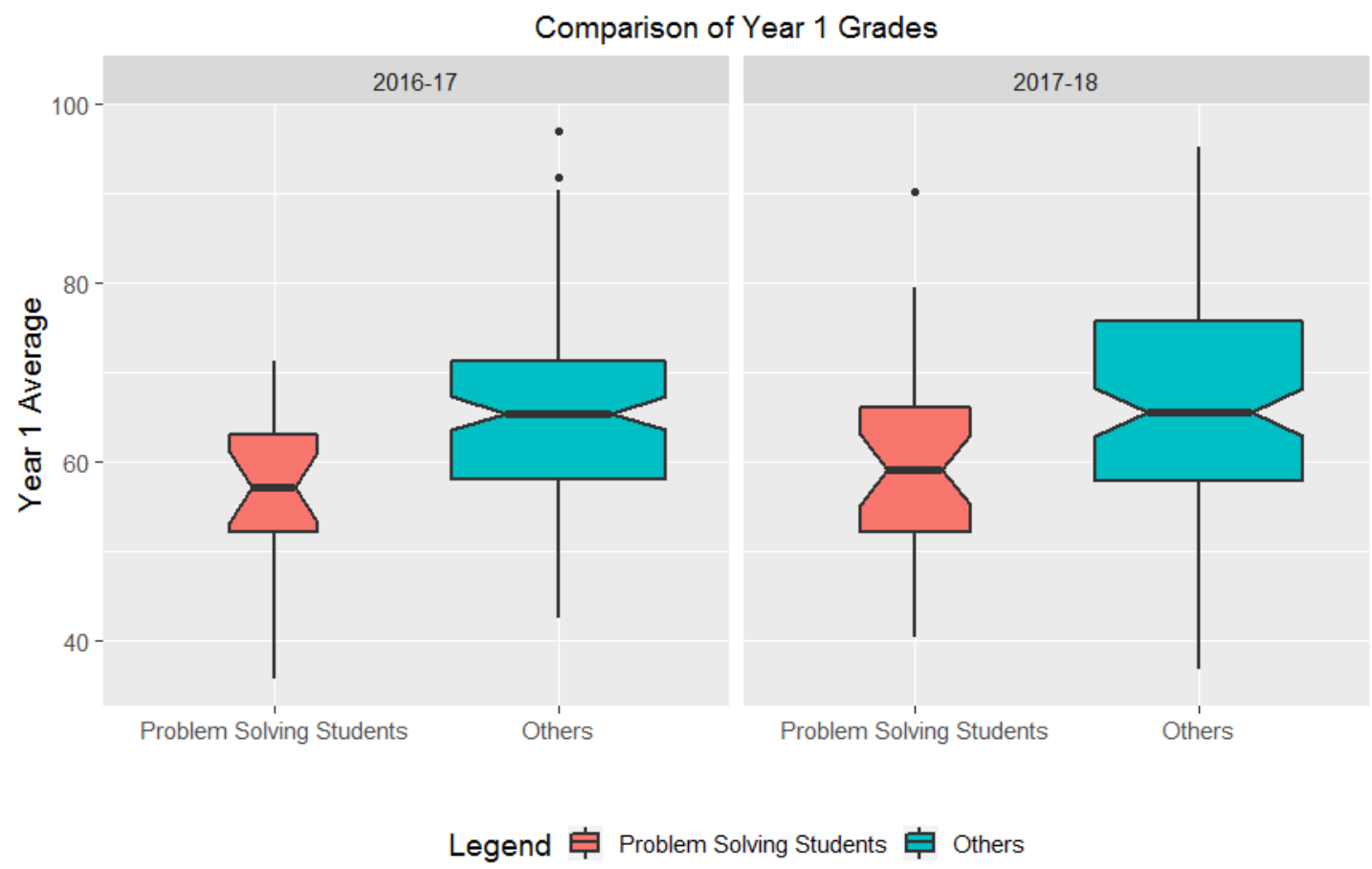

Figure 1: Year 1 grades for 2016-17 and 2017-18, comparing students who chose Problem Solving to the rest of their cohort.

The fact that a large number of apparently weaker students have been choosing Problem Solving was unexpected, though may present a useful opportunity as these students could potentially benefit the most from a module with a significant focus on mathematical thinking, independence and original work. However, there is a risk that if this continues, the module may be viewed by both students and academics as a 'soft' subject, that can be chosen in order to avoid taking a more challenging subject in year 2 - thus we may face the task of convincing students who perform well in traditional examinations to take a module that lies outside their comfort-zone.

Student feedback has so far been overwhelmingly positive (Table 1), though only a small number of students responded. We initially feared that open-ended nature of the problems and assessment tasks would be intimidating for students, however it would appear that many students enjoy having the flexibility to explore questions that they set themselves. The responses suggest that students also appreciate the value of a module that develops their employability skills. 
Table 1: Module Evaluations for Problem Solving, selected questions

STATEMENT

$\begin{array}{cc}\% \text { AGREE } & \% \text { AGREE } \\ 2016-17(N=10) & 2017-18(N=6)\end{array}$

\begin{tabular}{|c|c|c|}
\hline $\begin{array}{l}\text { The module has helped my personal development by } \\
\text { improving my employability skills (e.g. Presentation skills, } \\
\text { communication skills, reasoning skills, independent } \\
\text { learning). }\end{array}$ & 100 & 100 \\
\hline $\begin{array}{l}\text { The module inspired interest and was intellectually } \\
\text { stimulating. }\end{array}$ & 90 & 100 \\
\hline $\begin{array}{l}\text { I had a clear sense of what was required of me in the } \\
\text { assessment. }\end{array}$ & 100 & 100 \\
\hline Overall I am satisfied with this module. & 100 & 100 \\
\hline
\end{tabular}

Despite this positive student feedback, it quickly became apparent to the lecturer that the volume of summative coursework during the pilot year was excessive. While most groups could make a reasonable attempt at each week's problem, students were unhappy with the workload involved. More importantly, there was insufficient time for many of the activities that we wanted to promote, such as reviewing and evaluating solutions, or looking for generalisations, extensions and applications of their work.

Therefore, during the second iteration of Problem Solving, it was decided that the work submitted at the end of each class would be purely formative, and of the problems attempted during these classes, students would choose one from the first half of the semester and one from the second half, and prepare detailed written reports on each of these, worth $20 \%$ and $50 \%$ respectively. The group presentations would now be worth $30 \%$. The quality of submitted coursework appears to have improved significantly as a result of these changes.

In summary, Problem Solving in its current form appears to be working as intended - some of the coursework submitted, particularly in 2017-18, has contained genuinely impressive, original mathematical work. Nonetheless, some aspects will need to be reviewed over future iterations of the module. In particular, certain problems need to be reworked or replaced, and ensuring that student work is being assessed reliably and consistently is an ongoing challenge.

We hope that eventually, all second-year mathematics students will take this module, though some changes would be needed to make this possible. The three-hour tutorial style classes could not be taught by a single lecturer, nor could these take place in a traditional lecture hall. Even with a relatively small number of students, a significant amount of time is spent on assessment and feedback, as well as meeting groups of students during office hours. However, we would prefer to see the current format of this module remain broadly unchanged.

\section{References}

Badger, M., Sangwin, C.J., Hawkes, T.O., Burn, R.P., Mason, J., and Pope, S., 2012. Teaching problem-solving in undergraduate mathematics. Coventry University. Available at: http://www.mathcentre.ac.uk/resources/uploaded/guide.pdf [Accessed 11 March 2019]. 
Challis, N., Houston, K., and Stirling, D., 2003. Supporting Good Practice in Assessment in Mathematics, Statistics and Operational Research. Birmingham: Maths, Stats \& OR Network. Available at: https://www.heacademy.ac.uk/system/files/supportinggoodpractice.pdf [Accessed 11 March 2019].

Croft, T., and Grove, M., 2015. Progression within mathematics degree programmes. Grove, M., Croft, T., Kyle, J. and Lawson, D. [eds.]. Transitions in Undergraduate Mathematics. Birmingham: University of Birmingham, pp.173-189.

Hirst, K., and Biggs, N., 1969. Undergraduate projects in mathematics. Educational Studies in Mathematics, 1(3), pp.252-261. https://doi.org/10.1007/BF00558311

Iannone, P., and Simpson, A. [eds.], 2012. A Survey of Current Assessment Practices. In: Mapping university mathematics assessment practices. Norwich: University of East Anglia.

Mason, J., 2000. Asking mathematical questions mathematically. International journal of mathematical Education in Science and Technology, 31(1), pp.97-111. https://doi.org/10.1080/002073900287426

Mason, J., Burton, L., and Stacey, K., 2011. Thinking mathematically. London: Pearson Higher Education.

Polya, G., 1945. How to solve it: A new aspect of mathematical method. Princeton University Press.

Quality Assurance Agency (QAA), 2015. Subject Benchmark Statement: Mathematics, Statistics and Operational Research.

Rowlett, P. [ed.], 2011. HE Mathematics Curriculum Summit. Birmingham: MSOR Network.

Selden, J., Selden, A., and Mason, A., 1994. Even Good Calculus Students Can't Solve Nonroutine Problems. In: Kaput, J. \& Dubinsky, E., [eds.]. Research Issues in Undergraduate Mathematics Learning (MAA Notes Number 33). Washington DC: The Mathematical Association of America.

University of Bristol, 2016. Industrial Problem Solving in Higher Education. Available at: https://wikis.bris.ac.uk/display/ipshe/Home [Accessed 18 October 2018].

Waldock, J. [ed.], 2011. Developing Graduate Skills in HE Mathematics Programmes. Birmingham: National HE STEM Programme. Available at:

http://www.mathcentre.ac.uk/resources/uploaded/gradskills.pdf [Accessed 11 March 2019]. 